\title{
Wiring Stability of the Adult Drosophila Olfactory Circuit after Lesion
}

\author{
Daniela Berdnik, ${ }^{1}$ Takahiro Chihara, ${ }^{1}$ Africa Couto, ${ }^{2}$ and Liqun Luo ${ }^{1}$ \\ ${ }^{1}$ Howard Hughes Medical Institute, Department of Biological Sciences, Stanford University, Stanford, California 94305, and ${ }^{2}$ Research Institute of \\ Molecular Pathology, 1030 Vienna, Austria
}

\begin{abstract}
Neuronal wiring plasticity in response to experience or injury has been reported in many parts of the adult nervous system. For instance, visual or somatosensory cortical maps can reorganize significantly in response to peripheral lesions, yet a certain degree of stability is essential for neuronal circuits to perform their dedicated functions. Previous studies on lesion-induced neuronal reorganization have primarily focused on systems that use continuous neural maps. Here, we assess wiring plasticity in a discrete neural map represented by the adult Drosophila olfactory circuit. Using conditional expression of toxins, we genetically ablated specific classes of neurons and examined the consequences on their synaptic partners or neighboring classes in the adult antennal lobe. We find no alteration of connection specificity between olfactory receptor neurons (ORNs) and their postsynaptic targets, the projection neurons (PNs). Ablating an ORN class maintains PN dendrites within their glomerular borders, and ORN axons normally innervating an adjacent target do not expand. Likewise, ablating PN classes does not alter their partner ORN axon connectivity. Interestingly, an increase in the contralateral ORN axon terminal density occurs in response to the removal of competing ipsilateral ORNs. Therefore, plasticity in this circuit can occur but is confined within a glomerulus, thereby retaining the wiring specificity of ORNs and PNs. We conclude that, although adult olfactory neurons can undergo plastic changes in response to the loss of competition, the olfactory circuit overall is extremely stable in preserving segregated information channels in this discrete map.
\end{abstract}

Key words: plasticity; olfaction; cell ablation; glomerulus; olfactory receptor neurons; projection neurons

\section{Introduction}

Neuronal circuits can alter wiring in response to experience, learning, and injury. Such plasticity includes changes at different levels, from adjusting synaptic strength and making or breaking synapses to large-scale structural reorganization involving sprouting or pruning of axonal and dendritic branches. Examples of wiring plasticity have been documented during critical periods of neuronal circuit development, for example, in the mammalian visual system (Wiesel, 1982).

Numerous studies have demonstrated that, in adult animals, profound changes in neuronal connections in sensory and motor systems occur in response to peripheral injuries (Kaas, 1991; Buonomano and Merzenich, 1998). For example, Pons et al. (1991) described a massive long-term reorganization in the somatosensory cortex after deafferentation of an arm: the primary sensory cortex that previously represented the arm could respond

\footnotetext{
Received Nov. 18, 2005; revised Feb. 8, 2006; accepted Feb. 12, 2006.

This work was supported by a Human Frontier Science Program Organization long-term postdoctoral fellowship (D.B.), a Japan Science and Technology Overseas and Japan Society for the Promotion of Science for Research Abroad Fellowship (T.C.), and National Institutes of Health Grant R01-DC005982 (L.L.). L.L. is a Howard Hughes Medical Institute investigator. We thank T. Clandinin for the suggestion of the unilateral antenna removal experiment; B. Dickson for sharing results before publication; B. Tasic, T. Clandinin, B. Dickson, E. Hoopfer, T. Komiyama, L. Sweeney, and C. Darian-Smith for helpful comments on this manuscript; D. Luginbuhl for generating transgenic flies; and K. O'Kane, L. Stevens, M. Metzstein, M. Krasnow, E. Buchner, and Bloomington Stock Center for reagents.

Correspondence should be addressed to Liqun Luo, Howard Hughes Medical Institute, Department of Biological Sciences, Stanford University, Stanford, CA 94305. E-mail: Iluo@stanford.edu.

DOI:10.1523/JNEUROSCI.4941-05.2006

Copyright $\odot 2006$ Society for Neuroscience $\quad$ 0270-6474/06/263367-10\$15.00/0
}

to face stimuli. Gilbert and Wiesel (1992) showed that, after retinal lesion, visual cortical neurons representing the area of lesion have remarkable recovery of visual activity after initial silencing. The extent of plasticity and the underlying mechanisms are still under debate (Lund et al., 1994; Smirnakis et al., 2005). It is likely that short-term plasticity involves changes in the strength of existing connections, whereas long-term plasticity involves axonal sprouting to form new connections. Indeed, axonal sprouting in central (Darian-Smith and Gilbert, 1994; Florence et al., 1998) and peripheral (Darian-Smith and Brown, 2000; Darian-Smith, 2004) sensory systems in response to peripheral lesions have been reported. Dendritic reorganization also occurs in response to sensory denervation (Hickmott and Steen, 2005).

Here we test whether and to what extent structural reorganization occurs in the adult Drosophila olfactory system in response to injury. Olfactory systems differ from continuous maps used by the visual and somatosensory systems. From insects to mammals, olfactory receptor neurons (ORNs) expressing a given odorant receptor (OR) converge their axonal projections onto a single glomerulus in the antennal lobe/olfactory bulb (Axel, 1995; Vosshall, 2000). Olfactory information is subsequently relayed by projection neurons ( $\mathrm{PNs}$ ), each sending dendrites into a single glomerulus and its axons to higher brain centers (see Fig. $1 A$ ). The Drosophila olfactory system offers several experimental advantages for testing wiring plasticity. First, it is numerically simpler. Second, individual glomeruli are identifiable based on their stereotyped size, shape, and relative position (Laissue et al., 
1999). Third, unlike mammalian ORNs that are continuously born throughout life, all Drosophila ORNs are born and wiring of the olfactory circuit is completed during pupal development. Therefore, the assembly of neuronal connections during development and their maintenance in adults are two clearly separable phases. Last, genetic tools can be used to ablate and visualize specific classes of ORNs, PNs, or both.

We find that, although adult olfactory neurons can undergo plastic changes in response to loss of competition, the connection specificity of ORNs and PNs is extremely stable. Neither ORN axons nor $\mathrm{PN}$ dendrites sprout into neighboring glomeruli when their neighbors or partners are ablated. Hence, the muddling of information channels in this discrete map is prevented. We discuss possible mechanisms and the biological significance of this wiring stability.

\section{Materials and Methods}

Fly stocks. We used the following Gal4 drivers for either labeling or killing neurons: $y w$; Gal4-Mz19 UAS-mCD8GFP (see Figs. $1 B, 2 E, F, 4 D-F$, $6 A, B, 7), y w$; Gal4-GH146 UAS-mCD8GFP (see Fig. 6C-F) specific for subsets of PNs (Stocker et al., 1997; Jefferis et al., 2004); w; Or88aGal4/ $C y O, w$; Or $47 b G a l 4 / C y O$ (see Figs. $2 C, D, 3-5,7,8$ ) positive for two distinct antenna ORN classes, and w; Or $46 a$ Gal4/CyO (see Figs. $4 B, C, 8$ ) as a maxillary palp-specific ORN class (Komiyama et al., 2004).

We used $y w h s$-Flp (or $h s-F l p^{122}$ ) UAS-mCD8GFP to express Flp recombinase and label cell bodies with its neurites. We used HAsynaptotagmin as a marker for presynaptic termini (see Fig. $2 C, D$ ) (Robinson et al., 2002). The more active hs-Flp ${ }^{122}$ insertion (gift from M. Metzstein and M. Krasnow, Stanford University, Stanford, CA) was used to achieve complete activation of ricin toxin A (RTA) expression.

For cell ablation with RTA, we used either $w$; UAS-FRT- $w^{+} F R T$ $R T A^{1.1} / C y O$ (see Fig. $2 F$ ) or $w$; UAS-FRT- $w^{+} F R T-R T A^{19} / T M 3, S b$ (see Fig. 3) (Smith et al., 1996); for cell ablation with diphtheria toxin (DTI), we used either $w$; UAS-DTI ${ }^{18} / C y O$ (see Figs. $2 D, 5$ ) or $w$; UAS-DTI ${ }^{14} /$ TM3, Sb (see Figs. 6, 7) (Han et al., 2000). To repress DTI expression during development, we used either $w$; tubGal80ts ${ }^{\sharp 2 O}$; TM2/TM6b, Tb (see Fig. 6) or w; Sco/CyO; tubGal80ts ${ }^{\# 7}$ (see Fig. 7) (McGuire et al., 2004).

Generation of the line Mz19mCD8GFP. To create a stock that labels Mz19-Gal4-positive PNs without using the Gal4-UAS system, we used the "transposon swap" strategy (Sepp and Auld, 1999). First, we constructed a new enhancer trap vector by replacing Gal4 and the mini white gene in the pGalW enhancer trap vector with mCD8GFP and the yellow gene, respectively (Gerlitz et al., 2002) (T. Chihara and L. Luo, unpublished data). Transgenic flies were generated according to standard procedures. Then, $\mathrm{P}[\mathrm{Gal} 4, \mathrm{w}+]$ from the Mz19-Gal4 locus on the second chromosome was swapped with $\mathrm{P}[\mathrm{mCD} 8 \mathrm{GFP}, \mathrm{y}+]$ on the $\mathrm{X}$ chromosome. We selected male flies negative for the white gene and positive for the yellow gene in a $y w$ background (Sepp and Auld, 1999) and screened their brains for the presence of green fluorescent protein (GFP) signals in Mz19-positive PNs of the antennal lobe.

Or88a-CD2 and Or10a-CD2 constructs and transgenes. The rat CD2 coding region (Dunin-Borkowski and Brown, 1995) was cloned downstream of Drosophila Or88a and Or10a promoter fragments, respectively. The following primer sequences were used to amplify promoter elements from Drosophila genomic DNA: Or88a forward, 5' -(ATAAGAATGCGGCCGC)AATTCAATCTCAACCTTCTTTCCGATG-3'; Or88a reverse, 5' -(TATCAAATGCGGCCGC)ACTTAACTTTTCTGTATGGCTTCTACTGTCTGAAC-3'; Or10a forward, 5' -(ATAAGAATGCGGCCGC) GAAACGAAAGTAAATAAGCCCCAAAAA-3'; and Or10a reverse, 5' -(TATCAAATGCGGCCGC)GGATATAACTATGTGAACGACATCCTGGCAAG-3'. Transgenic flies were generated following standard procedures. Third chromosome insertions of Or88a-CD2 and Or47b$C D 2$ were each recombined with Or10a-CD2.

Cell ablation protocols. Heat-shock (hs) induction in RTA cell ablation experiments (see Figs. $2 F, 3$ ) was started during pupal development at $44 \mathrm{~h}$ after puparium formation (APF) for Or47bGal4, $65 \mathrm{~h}$ APF for Or88aGal4, and $48 \mathrm{~h}$ APF for Gal4-Mz19. Heat shocks at $37^{\circ} \mathrm{C}$ were repeated three times a day for $30 \mathrm{~min}$ each for 14-16 consecutive days, and pupae/flies were otherwise kept at $25^{\circ} \mathrm{C}$. Axons and dendrites can no longer be detected by day 7 after eclosure, leaving at least $7 \mathrm{~d}$ to allow for reorganization. To assess long-term effects on neurons, we waited $30 \mathrm{~d}$ after cell ablation was complete.

UAS-DTI was induced directly from Or-Gal4 drivers by raising adult flies at $29^{\circ} \mathrm{C}$ for $12 \mathrm{~d}$ before dissection (see Fig. 5). ORN cell bodies and their axons were undetectable $5 \mathrm{~d}$ after emerging. This leaves an additional window of $7 \mathrm{~d}$ for possible reorganization to occur. For PN ablations, using Gal4-Mz19 and Gal4-GH146, DTI production was developmentally controlled via tubGal80ts. Flies were raised at $18^{\circ} \mathrm{C}$ until they eclosed and shifted to $29^{\circ} \mathrm{C}$ for the first $12 \mathrm{~d}$ (see Fig. 6) or $8 \mathrm{~d}$ (see Fig. 7) of adulthood, respectively, before they were dissected. After $3 \mathrm{~d}$ of temperature shift, ORN cell bodies and projections were eliminated, whereas in the control (no UAS-DTI, but tubGal80ts was present), the complete Gal4 expression pattern was turned on. This leaves a time window for possible wiring plasticity of $9 \mathrm{~d}$ (see Fig. 6) or $5 \mathrm{~d}$ (see Fig. 7) after PN ablation or double PN and ORN ablation, respectively.

Immunostainings. The procedures for fixing, staining, and imaging along with antibodies used have been described previously (Zhu and Luo, 2004). Additionally, we used rabbit anti-GABA (Sigma, St. Louis, MO) at 1:500 and rabbit anti-HA at 1:1000 (Abcam, Cambridge, MA). We determined mean fluorescence intensities for the presynaptic marker nc82 and GABA of single representative confocal sections and calculated ratios between the glomeruli VA1d and DA4. To measure glomerular volumes, adult whole-mount brains of the same age and sex were stained with nc82 antibodies and imaged at $512 \times 512$ pixels in $1 \mu \mathrm{m}$ confocal sections on a Zeiss (Oberkochen, Germany) LSM510. The contours of glomeruli VA1d and DA4 were traced in individual sections. Volumes were calculated by summing individual areas multiplied by image thickness $(1 \mu \mathrm{m})$.

\section{Results}

\section{Experimental system}

The peripheral olfactory system of adult Drosophila contains $\sim 50$ input information channels. With few exceptions (Couto et al., 2005; Fishilevich and Vosshall, 2005; Goldman et al., 2005), each of the 1300 ORNs expresses 1 of the $\sim 50$ ORs (Clyne et al., 1999; Gao and Chess, 1999; Vosshall et al., 1999). ORNs expressing the same OR converge their axonal projections onto 1 of the $\sim 50$ glomeruli in the antennal lobe (Gao et al., 2000; Vosshall et al., 2000; Couto et al., 2005). Here, information is relayed to the second-order olfactory PNs, most of which send dendrites to a single glomerulus and axons to higher olfactory centers (Fig. 1A) (Jefferis et al., 2001). An important feature of this system is that most glomeruli in the antennal lobe are individually identifiable by size, shape, and position (Laissue et al., 1999), such that a general presynaptic marker allows us to distinguish individual glomeruli (Fig. $1 B$, nc82 staining in red).

In this study, we focused our analysis on three large-landmark glomeruli, VA1lm, VA1d, and DA1, that are adjacent to each other on the lateral, anterior surface of the antennal lobe (Fig. $1 B$, white rectangle). VA1lm and VAld are innervated by ORNs expressing Or47b and Or88a, respectively (Vosshall et al., 2000; Komiyama et al., 2004). The PN driver Gal4-Mz19 labels a subset of PNs sending their dendrites to DA1 and VA1d (Fig. $1 \mathrm{~B}$, green) along with a small posterior glomerulus DC3 (mostly masked by VAld in the $z$ projection of Fig. $1 B$ ) (Jefferis et al., 2004). Therefore, the use of these OR and PN promoters allows us to selectively label and ablate respective ORNs and PNs genetically.

These tools allowed us to perform a series of experiments and ask specific questions regarding neuronal reorganization in response to ablation (Fig. $1 C-E$ ). First, if we ablate an ORN class, what will happen to the dendritic projections of PNs that innervate the same glomerulus or an adjacent glomerulus (Fig. 1C)? Second, if we ablate an ORN class, what will happen to the axonal 

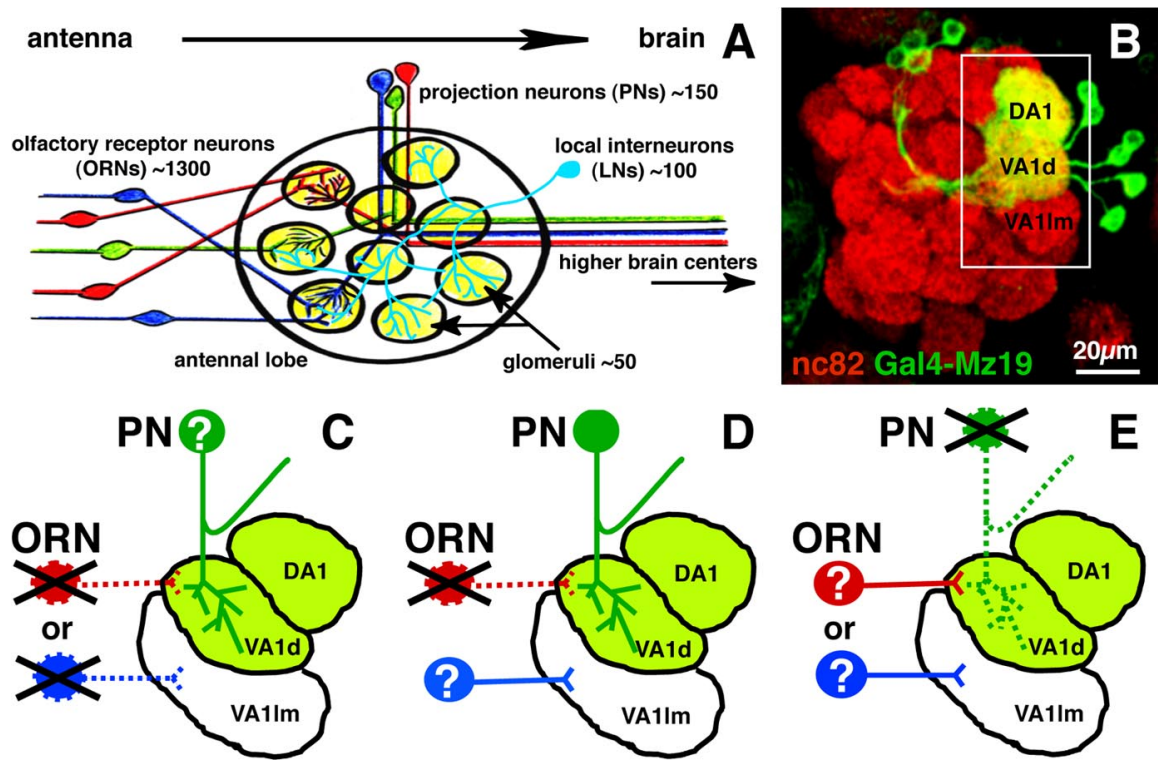

Figure 1. Schematic overview of cell ablation experiments. $A$, Schematic showing the organization of the Drosophila olfactory circuit. $\boldsymbol{B}$, The antennal lobe of the Drosophila adult brain is shown by staining using the nc 82 antibody (in red), which serves as a presynaptic marker. The three glomeruli that are the focus of this study are outlined by the white rectangle. Two of these three glomeruli are dendritic targets of second-order PNs expressing Gal4-Mz19 (green), which labels approximately six dorsal PNs (projecting to VA1d and DC3, a posterior glomerulus behind VA1d in this confocal $z$ stack) and approximately seven lateral PNs (projecting to DA1). Genotype: Gal4-Mz19 UAS-mCD8GFP. C-E, Schematics illustrating a series of cell ablation experiments that eliminate one specific class of olfactory receptor neurons (e.g., ORNs dotted line in red) while monitoring either the fate of its corresponding postsynaptic PNs (in green) (C) or ORNs projecting to the adjacent glomeruli (in blue) (D), or eliminate PNs (dotted line in green) and observe the fate of their presynaptic partners (ORNs in red) or neighbors (ORNs in blue) (E). Unless otherwise indicated, all images in this study are maximum-intensity z projections of $1 \mu \mathrm{m}$ spaced confocal stacks. Dorsal is up and the midline is to the left in each panel.

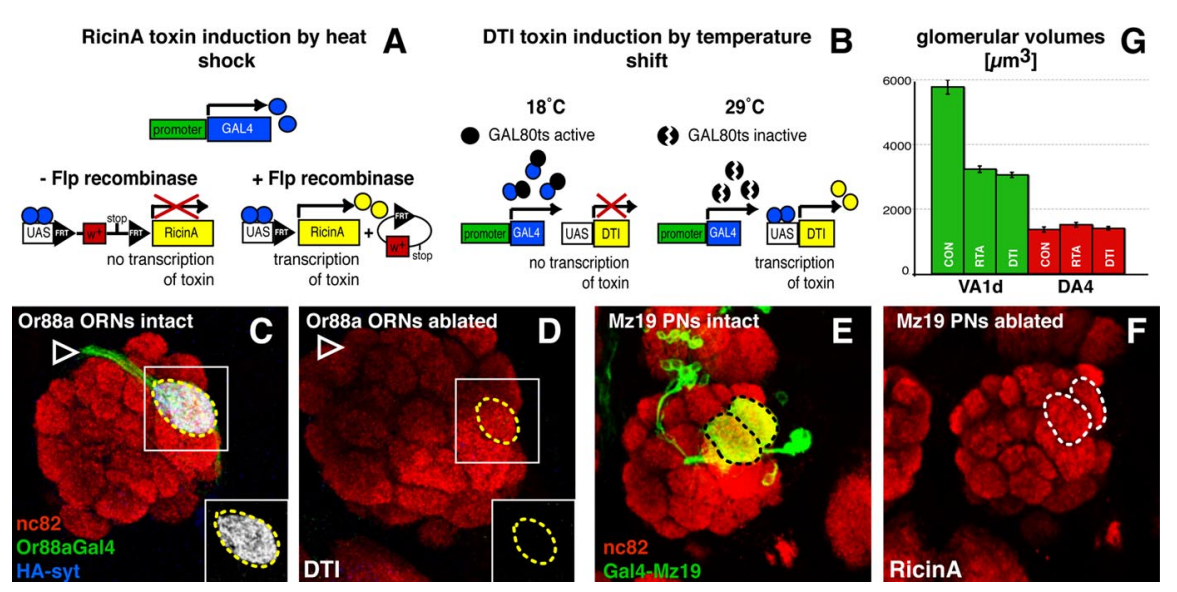

Figure 2. Cell-autonomous toxin expression causes neuronal death. $\boldsymbol{A}, \boldsymbol{B}$, Two different Gal4-UAS-based cell ablation methods are shown. RTA is turned on by driving Flp recombinase from a heat-shock-inducible promoter. This leads to the removal of a stop between two FRT sites, allowing Gal4-driven toxin transcription $(\boldsymbol{A})$. DTl induction is regulated with Gal80ts, which binds to Gal4 at $18^{\circ} \mathrm{C}$ preventing transcription; at $29^{\circ} \mathrm{C}$, Gal 80 becomes inactive and toxin is expressed $(\boldsymbol{B})$. C, D, Ablation of 0r88a-expressing ORNs using DTI. In the control, axon termini of Or88a-expressing ORNs (green) reside in VA1d (yellow circle) in which they form synapses (blue and inset) (C). Toxin expression results in the loss of presynaptic termini indicated by HA-synaptotagmin (inset) and degeneration of all axons, as indicated by the lack of green $(\boldsymbol{D})$ in VA1d (yellow circle) and its contralateral projections to the opposite antennal lobe (arrowhead). $\boldsymbol{E}, \boldsymbol{F}$, Ablation of Mz19-positive PNs using RTA. In the control, Mz19-positive dendrites (green) innervate the glomeruli VA1d and DA1 (black circles) (E). RTA effectively kills PNs shown by the absence of GFP-labeled PN cell bodies and dendrites $(\boldsymbol{F})$. G, Quantification of glomerular volumes resulting from VA1d ORN ablation. Volumes of ORN axon-free VA1d glomeruli (green bars) are reduced by $>40 \%$ for RTA- and DTI-induced cell ablations, whereas the volume of the adjacent and unperturbed glomerulus DA4 remain unchanged (red bars). $n=7-9$ glomeruli. Error bars indicate SEM. Genotypes: $y w h s-F l p$ UAS-mCD8GFP/yw UAS-HA-syt; Or88a-Gal/CyO (C), (CON in G); yw hs-Flp UASmCD8GFP/yw UAS-HA-syt; Or88a-Gal4 UAS-DTI/CyO (D) (DTI in G); yw hs-Flp ${ }^{122}$ UAS-mCD8GFP; Gal4-Mz19 UAS-mCD8GFP/Cy0 (E); yw hs-Flp ${ }^{122}$ UAS-mCD8GFP; Gal4-Mz19 UAS$m$ CD8GFP/UAS-FRT $w+$ FRT-RTA $(\boldsymbol{F})$; and $y w$ hs-Flp ${ }^{122}$ UAS-mCD8GFP; Or88aGal4/UAS-FRT $w+$ FRT-RTA (RTA in G). projections of ORNs normally innervating an adjacent glomerulus (Fig. $1 D)$ ? Finally, if we ablate specific PN classes, what will happen to ORN axons projecting to the same glomerulus or an adjacent glomerulus (Fig. 1E)?

Regulated toxin expression effectively ablates specific neuronal classes

We used two strategies to achieve cellautonomous ablation without compromising the viability of the animals. In the first strategy, we used transgenic flies that allow for tissue-specific expression of the RTA chain under Gal4 control only during heat-shock-mediated Flp recombinase induction (Smith et al., 1996). The recombination in cis between two FRT (Flp recombinase target) sites, which flank a transcription stop and lie between RTA and the promoter, leads to the excision of the stop and consequent expression of the toxin (Fig. 2A). This strategy offers temporal control of cell ablation and avoids lethality, which could result from expression of RTA during development.

As a second ablation strategy, we used a transgene containing the A chain of DTI preceded by an upstream activating sequence (UAS) (Han et al., 2000). To avoid lethality in combination with some Gal4 lines (e.g., Gal4-Mz19) and to gain temporal control of toxin expression, we used a transgene encoding a temperaturesensitive Gal80 (Gal80ts) (McGuire et al., 2004). The animals were reared at permissive temperature during development, and adults were shifted to restrictive temperature to inactivate Gal80 and permit toxin expression (Fig. $2 B$ ).

To examine adult wiring plasticity, we ablated ORNs and PNs after they have already established their neuronal connections. In fact, ablation before connection formation was not possible because of experimental constraints. Specifically, Drosophila OR expression is not detectable until ORN axon targeting is essentially completed and targeting specificity is achieved. For example, Or88a-Gal4driven mCD8GFP expression is not detectable until 65 h APF (data not shown). This timing of OR expression is significantly delayed compared with the maturation of adult-like glomeruli in the antennal lobe at $50 \mathrm{~h}$ APF (Jefferis et al., 2004). Or47b-Gal4 is the first detectable OR driver, with onset of reporter gene expression at $44 \mathrm{~h}$ APF (data not shown). Because we rely on OR-Gal4 to drive toxin expression in both strategies (Fig. 2A,B), we could only ablate the corresponding ORNs after the initial wiring was com- 
pleted. Likewise, all PN ablation experiments were performed after the initial PN targeting was completed because $\mathrm{PN}$ driver-mediated toxin expression during development leads to lethality (see below).

We optimized protocols for each combination of toxin and cell types to achieve complete ablation of intended cell types (see Materials and Methods). Figure 2C-F shows two examples for DTI ablation of ORNs and RTA ablation of PNs. In the first example, we ablated Or88a-expressing ORNs, which send their axons to VAld glomeruli in both the ipsilateral and contralateral antennal lobes (Fig. 2C). Induction of DTI led to ORN death (see below), loss of presynaptic terminals (Fig. 2C,D, compare insets) and axons in the corresponding glomerulus, and loss of its contralateral projections (Fig. $2 D$, yellow circle and arrowhead, respectively). Ablation of an entire ORN class also results in a significant reduction of the corresponding glomerular size (Fig. 2, compare circles in $D$ with $C$; quantified in $G$ ).

In the second example, we used Gal4Mz19 to ablate $\sim 13$ PNs, including the PNs innervating DA1 and VA1d (Fig. 2E, black circles). After RTA expression, all Mz19-positive PNs were ablated and their dendrites were absent from the glomeruli (Fig. $2 \mathrm{~F}$, white circles), as shown by a complete lack of GFP staining.

Unless otherwise mentioned, we used the two methods for cell ablation described above (Fig. $2 A, B$ ) in parallel. For all experiments in which a single neuronal class was eliminated, we assessed the effects of the elimination on other neurons at least $7 \mathrm{~d}$ and for some as long as $30 \mathrm{~d}$ after cells were completely ablated. We obtained similar results using either RTA or DTI for each experiment (summarized in supplemental Table 1, available at www.jneurosci.org as supplemental material).

\section{Examination of PN dendrites after ORN ablation}

To study the effect of ablations of single class ORNs on PN dendrites, we used Gal4 lines of Or88a and Or47b. Both Or88a and Or47b are expressed in 50-60 cell bodies per antenna (Fig. $3 A, E$ ). Or88a axons innervate VA1d (Figs. $2 C, 3 B$ ), and Or47b axons target the adjacent VAllm (Fig. $3 F$ ). In the same animals, we labeled a subset of PNs using an enhancer trap line Mz19mCD8GFP (green). This strain was generated by replacement of Gal4 with mCD8GFP via transposon swapping (see Materials and Methods), thus allowing the visualization of Mz19-positive PNs independent of the Gal4-UAS system.

Figure 3, $C$ and $G$, shows additional examples of effective ORN ablation after induction of ricin A expression. Even after overexposure (Fig. 3, compare $C, G$ with $A, E$ ), only few GFP-positive cells were barely detectable, and no axons were visible within the third antennal segments. No axons were detectable in the antennal lobe (Fig. $3 D, H$, open arrowheads, compared with $B, F$ ). The targeted glomeruli were smaller in size (Figs. 3, compare circles in $B$ with $D$ and $F$ with $H, 2 G$ ) but were still clearly distinguishable by nc82 staining despite the loss of all ORN axons.

What happens to the $\mathrm{PN}$ dendrites after their presynaptic ORNs are ablated? A priori, we conceived several possibilities:
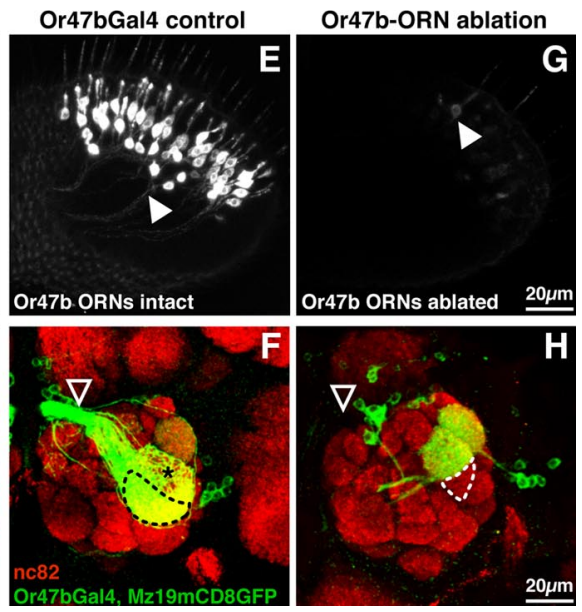
G
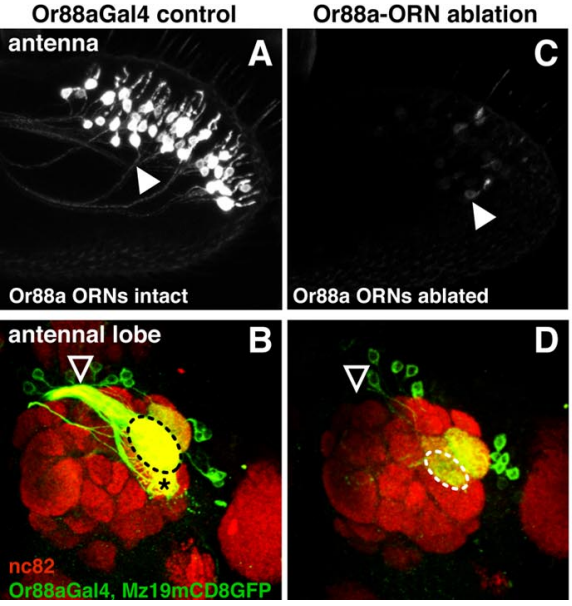

Or

Figure 3. Examination of PNs after ORN ablation. $A, B$, In the control, Or88a is expressed in $\sim 50-600$ RNs located in the adult third antennal segment $(\boldsymbol{A})$. Their axons (green) project into glomerulus VA1d (black circle), which is also positive for Mz19-labeled with the strong 0r-Gal4 lines in one image. The appearance of ORN axons spilling into adjacent glomeruli (asterisks in $\boldsymbol{B}$ and $\boldsymbol{F}$ ) is caused by axon accumulation at the surface of the adjacent glomeruli before entering into the correct glomerulus.

PNs might withdraw from the glomerulus for the lack of presynaptic partners that stabilize synaptic connections; they might invade neighboring glomeruli to establish new connections; or these PNs might altogether die for lack of innervation. However, after Or88a ORNs (projecting to VA1d) were ablated, we found that Mz19-positive PN dendrites remained within the borders of the VAld glomerulus despite the complete lack of ORN axons (Fig. 3D) (supplemental Table 1, available at www.jneurosci.org as supplemental material). They did not invade neighboring glomeruli (e.g., VA1lm).

In a separate experiment, we ablated the VAllm ORNs using Or47b-Gal4 (Fig. 3, compare G, E). Mz19 PN dendrites remained confined to their target glomeruli after ORN axons from the adjacent glomerulus were removed (Fig. 3H) (supplemental Table 1, available at www.jneurosci.org as supplemental material). Thus, ablation of single ORN classes does not alter the dendritic innervation pattern of PNs normally projecting to the same or a neighboring glomerulus. In addition, PN dendrites remained at their correct glomerular targets for up to $30 \mathrm{~d}$ after any of the two ORN classes had been ablated (see brackets in supplemental Table 1, available at www.jneurosci.org as supplemental material).

\section{PN dendrite stability after many ORN classes are ablated}

To extend the above finding, we examined the effects of ORN elimination on PN dendrite stability by ablating many ORN classes. Approximately $90 \%(\sim 1200)$ of all ORN cell bodies are located in the third antennal segment (Fig. $4 A$, red), whereas the remaining $10 \%(\sim 120)$ are found in the maxillary palps (Fig. $4 A$, blue) (Stocker, 1994). Cutting off the third antennal segments on both sides should result in ablation of all ORNs that reside in the antennae but not those in the maxillary palps. To verify specificity of this procedure, we used two Or-Gal4 lines crossed to UASmCD8GFP to label axons from two ORN classes. Or88a ORNs reside in the antenna and project their axons to VAld (circle), 

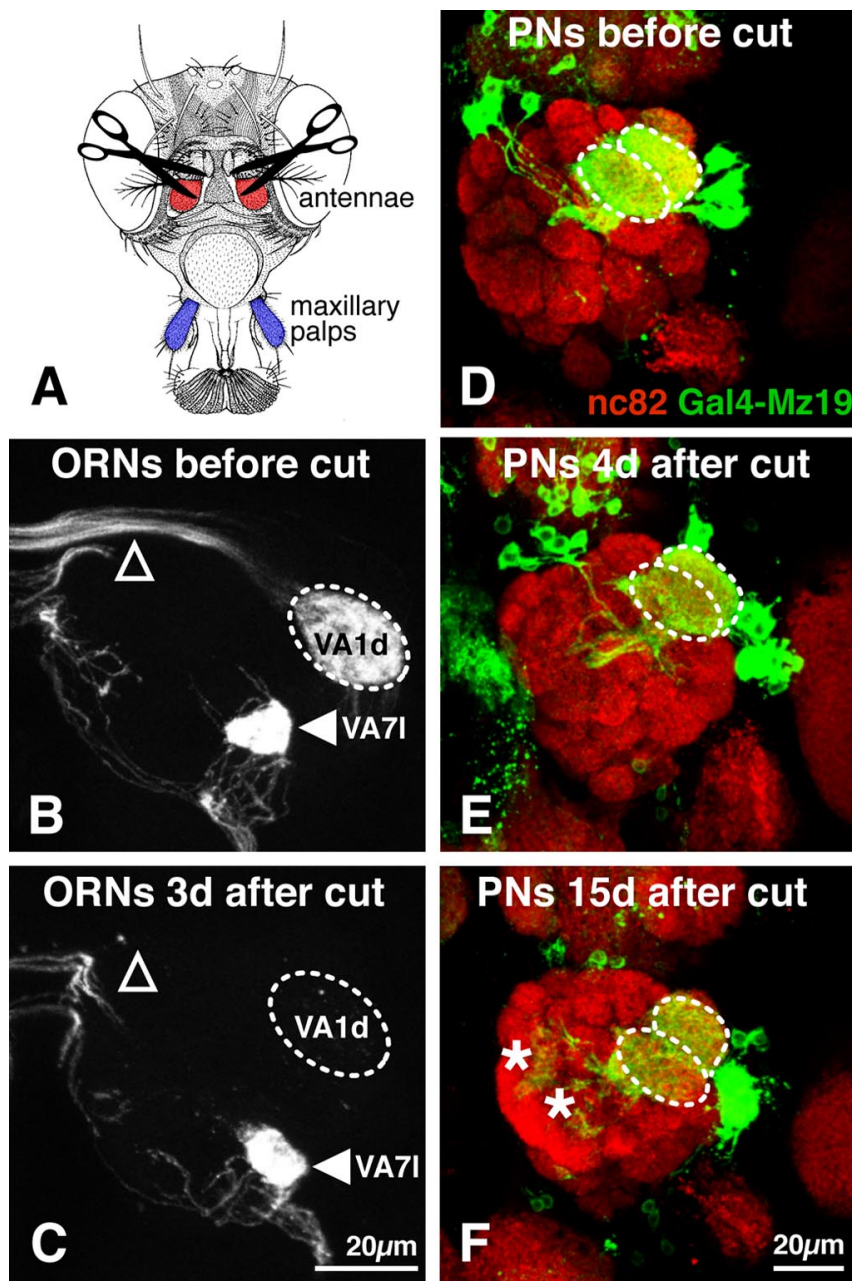

Figure 4. Examination of PNs after antennae removal. $A, O R N$ s are removed by cutting off both third antennal segments (red) from the head leaving maxillary palp ORNs (blue) intact. $\boldsymbol{B}$, C, Antenna ORNs expressing Or88a target axons to glomerulus VA1d (white circle), whereas maxillary palp ORNs expressing 0r46a target to the ventral glomerulus VA7I (filled arrowhead) (B). Three days after antennae removal, Or88a axons in VA1d (white circle) and their contralateral axonal projections (open arrowheads) are not detectable (C). VA7I axons remain undisturbed (filled arrowhead). Genotype in B and C: yw UAS-mCD8GFP/+; Or88aGal4/0r46aGal4. D-F, Mz19-labeled PN dendrites invade two superficial glomeruli, DA1 and VA1d (white circle) (D). Four days after antennae removal, Mz19-labeled PN dendrites still innervate their correct glomeruli $(\boldsymbol{E})$. Even $15 \mathrm{~d}$ after surgery, dendrites stay at their correct glomeruli $(\boldsymbol{F})$. Green is Mz19-driven mCD8GFP, and red is nc82. Genotype: $y w$; Gal4-Mz19 UAS-mCD8GFP.

whereas the maxillary palp Or46a ORN axons innervate VA71 (Couto et al., 2005) (Fig. 4B, filled arrowhead). At $72 \mathrm{~h}$ after antennae removal, all axons targeting VAld (circle) had degenerated (Fig. 4, compare $C$ with $B$ ), whereas axons projecting to VA7l persisted (Fig. $4 C$, filled arrowhead).

To test the dependence of PN dendrites on their presynaptic input, we used Gal4-Mz19 in combination with UAS-mCD8GFP to label a subset of PNs (green) invading three glomeruli that all receive sensory input from antennal ORNs (Fig. 4D). At $4 \mathrm{~d}$ after antennae removal from young animals, PN dendrites still remained at their correct glomeruli (VA1d and DA1 are encircled based on nc82 staining), although the axons of their partners had already degenerated by this time (Fig. $4 E$, circle). At $15 \mathrm{~d}$ after surgery, a time when all examined antennal axons have degenerated (Vosshall et al., 2000), the presynaptic marker nc82 pattern (Fig. $4 F$, red) was less discrete, presumably attributable to ablation of the majority of ORNs. Although we observed in 16 of 24 antennal lobes a few minor branches that sprouted into random areas within the antennal lobe (asterisks), the majority of PN dendrites still resided at their appropriate glomerular positions (Fig. 4F). Thus, PNs dendrites remain at their correct glomeruli even after the majority of ORN axons are ablated.

\section{Examination of ORN axons after ablation of ORNs projecting} to an adjacent glomerulus

In the mammalian cortex, peripheral lesions generate cortical areas that are devoid of sensory input. Later, these cortical areas can recover activity, likely via widespread expansions of axonal processes representing sensory input surrounding the lesion (see Introduction). It is therefore possible that, in the Drosophila olfactory circuit, adjacent ORN axons may invade the glomerulus in which the native ORN axons are ablated. This may also account for PN dendrite stability despite the loss of their natural partners.

To test the possibility of ORN axonal plasticity, we performed similar ORN ablation experiments, as documented in Figure 3, but focused our analysis on the ORN axons innervating a target adjacent to the ablated glomerulus (Fig. 5). As a control, we also monitored axons that originate from Or10a-expressing ORNs and project to a posterior glomerulus DL1 (Couto et al., 2005), which contacts neither VAld nor VAllm. To visualize ORN axons independently of the Gal4-UAS system, which we used for cell ablation, we used an existing Or47b-CD2 transgene (Zhu and Luo, 2004), in which the Or47b promoter directly drives the CD2 marker expression that labels ORN axons in VAllm (Fig. $5 A, A^{\prime}$ ). We also generated new transgenes Or88a-CD2 and Or10a-CD2, which label VA1d and DL1, respectively (Fig. $5 C, C^{\prime}$ ).

After Or88a ORNs were ablated (indicated by the lack of green signal within the circle in Fig. 5B), Or47b axons remained confined to the adjacent VAllm glomerulus (Fig. $5 B^{\prime}$, blue in $B$ ) (supplemental Table 1, available at www.jneurosci.org as supplemental material). Anti-CD2 antibody staining showed that Or47b axons neither spilled over into the ORN axon-depleted VAld glomerulus nor appeared destabilized, similar to the internal control, DL1 axons (Fig. 5A,B). Likewise, ablating Or47b axons did not cause Or88a axons to invade the adjacent ORN axon-free VA1lm glomerulus (Fig. 5D) (supplemental Table 1, available at www.jneurosci.org as supplemental material). These experiments indicate a lack of axonal sprouting when deafferented territories nearby become available, even after long-term sensory deprivation of up to $30 \mathrm{~d}$ (see brackets in supplemental Table 1, available at www.jneurosci.org as supplemental material).

\section{Examination of ORN axons after PN ablation}

So far, we have shown that PN dendrites are stable in the absence of their natural presynaptic partners, and their maintenance is not attributable to invasion of nearby ORN axons of a different class. These experiments demonstrate the extreme stability of PN dendrites in the adult olfactory circuit. Do PN dendrites serve to stabilize their presynaptic partners? If so, we would expect changes of their partner ORN axons when we ablate the PNs: these axons could withdraw or invade adjacent glomeruli to form new connections with dendrites of neighboring PN classes.

To address this question, we first performed cell ablation experiments in a small subset of PNs using Gal4-Mz19 and followed the fate of their presynaptic partners. Because Gal4-Mz19 is expressed during development in essential tissues such as the larval ventral nerve cord and muscles (data not shown), we used Gal80ts to suppress DTI transcription at a permissive tempera- 
ture $\left(18^{\circ} \mathrm{C}\right)$ until pupa eclose into adults (Fig. $2 B$ ). Then, we shifted to a restrictive temperature $\left(29^{\circ} \mathrm{C}\right)$ for 12 consecutive days before brains were dissected (Fig. 6). DTI expression led to PN death $3 \mathrm{~d}$ after rearing flies at $29^{\circ} \mathrm{C}$ (data not shown); thus, our protocol left $\sim 9 \mathrm{~d}$ to examine effects on ORN axons after PN ablation. PN death (lack of green staining in Fig. $6 B$ compared with $A$; also compare Fig. $2 E, F$ ) did not appear to alter the glomerular size, as indicated by nc82 staining. What happens to the ORN axons that innervate the same glomerulus?

As shown in Figure 6, $B$ and $B^{\prime}$, Or88a axons still properly innervated the VA1d glomerulus after Mz19-positive PNs had been ablated. Both methods of cell ablation yielded similar results (supplemental Table 1, available at www.jneurosci.org as supplemental material). This selective PN ablation did not affect ORN axons that project to Mz19-negative glomeruli, including the neighboring VA1lm (supplemental Table 1, available at www.jneurosci.org as supplemental material) and the posterior DL1 glomerulus (Fig. 6, compare $B, B^{\prime}$ with $\left.A, A^{\prime}\right)$. Thus, VAld PN dendrites do not appear to be required for the maintenance of axons of their partner ORNs or other ORNs.

We also used a second PN Gal4 driver GH146 (Stocker et al., 1997) that is expressed in the majority of PNs. Gal4GH146 combined with UAS-mCD8GFP (in green) labels $\sim 40$ of the $\sim 50$ glomeruli in the antennal lobe, including VAld, VA1lm, and DL1 (Fig. 6C,E). We used the same Gal80ts-DTI method (Fig. 2B) for ablations of GH146-positive PNs. Expression of DTI toxin effectively ablated GH146-labeled PNs in adults (see the loss of green signal in Fig. 6D,F). Despite the elimination of the majority of PNs, the glomerular structures of the antennal lobe remained essentially organized, with most individual glomeruli still recognizable by nc82 staining, including VA1d, VA1lm, and DL1 (Fig. 6D,F). Remarkably, ORN axons projecting to these three glomeruli remained intact and did not expand to neighboring glomeruli (Fig. 6D,F, blue, $\left.D^{\prime}, F^{\prime}\right)$. Together with the results from the Mz19-positive PN ablation, we conclude that, in the adult antennal lobe, PN dendrites are not essential for maintaining normal axon projections of their synaptic partners, the ORNs.

Double-ablation experiments and the contribution of local interneurons Ablation of neither ORNs alone nor PNs alone resulted in the elimination of the corresponding glomerulus (Figs. 2-6).
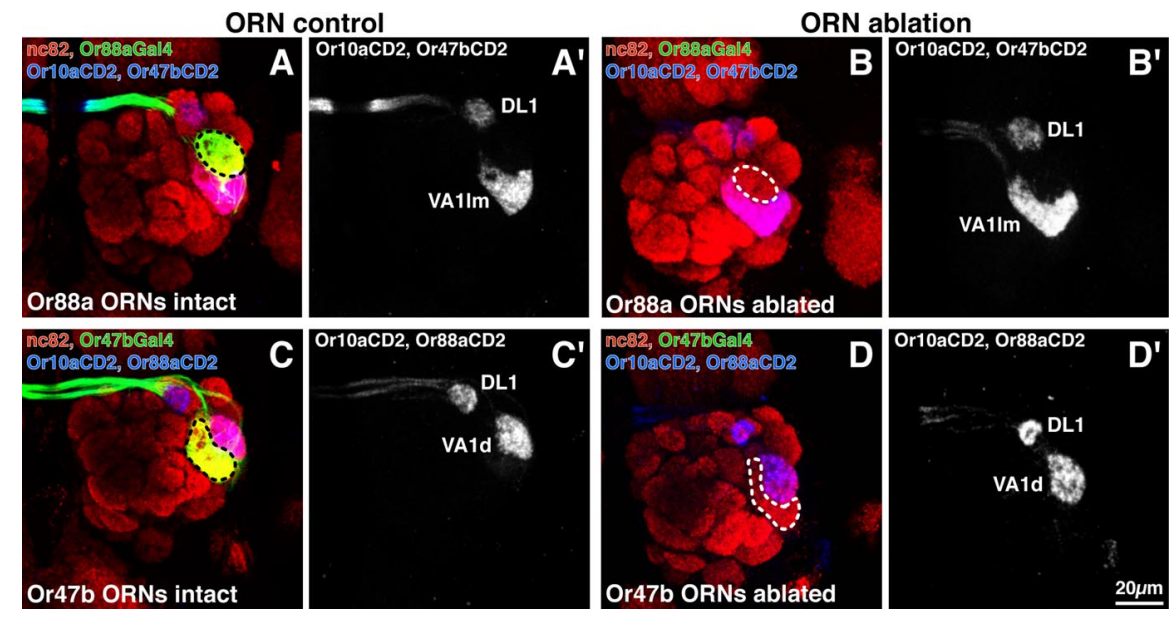

Figure 5. ORN axons do not invade adjacent axon-free glomeruli. $\boldsymbol{A}, \boldsymbol{B}$, Control $(\boldsymbol{A})$ and cell ablation $(\boldsymbol{B})$ of Or88a ORNs projecting to VA1d (circle, compare green signal in $\boldsymbol{B}$ with $\boldsymbol{A}$ ). ORN axons projecting to the adjacent (VA1/m) or another nonadjacent glomerulus (DL1) remain intact (compare blue in $\boldsymbol{B}$ with $\boldsymbol{A}$; or axon only staining in $\boldsymbol{B}^{\prime}$ with $\boldsymbol{A}^{\prime}$ ). $\boldsymbol{C}, \boldsymbol{D}$, Control $(\boldsymbol{C})$ and cell ablation (D) of Or47b ORNs projecting to VA1Im (circle, compare green signal in $\boldsymbol{D}$ with $\boldsymbol{C}$ ). ORN axons projecting to the adjacent glomerulus (VA1d) or another glomerulus a few glomeruli away (DL1) remain intact (compare blue in $\boldsymbol{D}$ with $\boldsymbol{C}$; or axon only staining in $\boldsymbol{D}^{\prime}$ with $\left.\boldsymbol{C}^{\prime}\right)$. Green is $\mathrm{m}\left(\mathrm{D} 8 \mathrm{GFP}\right.$, red is $n c 82$, and blue is $\left(D 2 . \boldsymbol{A}^{\prime}-\boldsymbol{D}^{\prime}\right.$ shows $(D 2$ only channels for $\boldsymbol{A}-\boldsymbol{D}$. Genotypes: $y \boldsymbol{W}$ hs-FIp UAS-mCD8GFP; Or88aGal4/Cy0; Or47b-CD2 Or10a-CD2/+ (A), yw hs-FIp UAS-mCD8GFP; Or88aGal4/Or88aGal4 UAS-DTI; Or47b-CD2 Or10a-CD2/+ (B), yw hs-Flp UAS-mCD8GFP; Or47bGal4/CyO; Or88a-CD2 Or10a-CD2/+ (C), and yw hs-Flp UASmCD8GFP; Or47bGal4/Or47bGal4 UAS-DTI; Or88a-CD2 Or10a-CD2/+ (D).
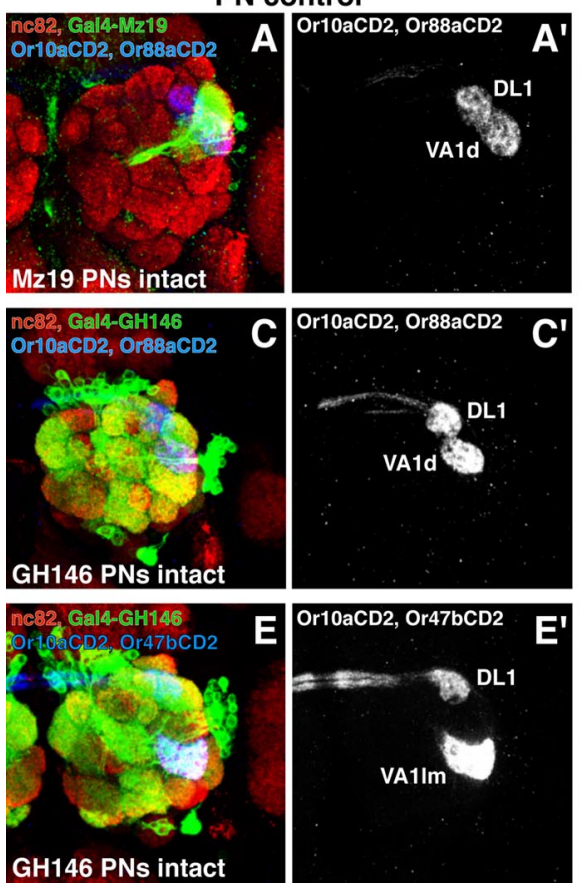

\section{PN control}

PN ablation

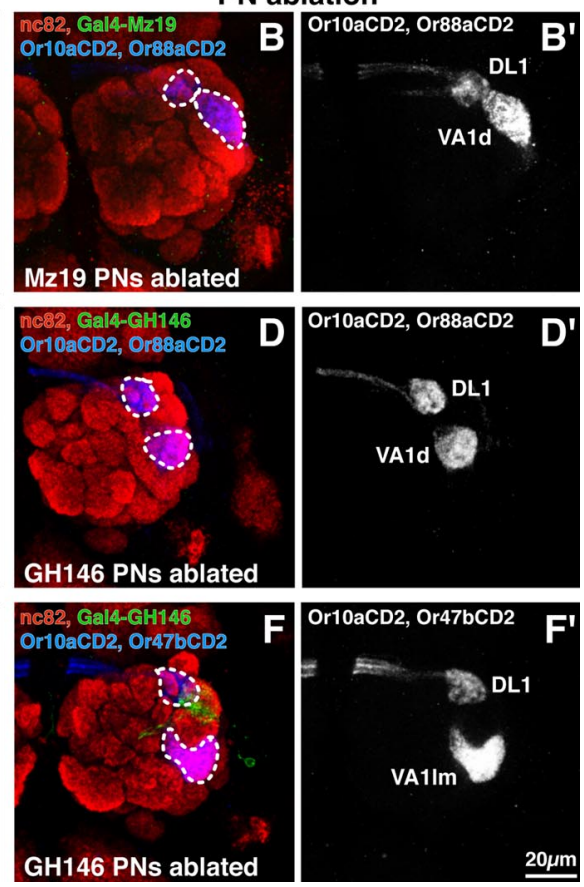

Figure 6. Examination of ORN axons after PN ablation. $\boldsymbol{A}, \boldsymbol{B}$, Control $(\boldsymbol{A})$ and cell ablation with DTI ( $\boldsymbol{B})$ of Gal4-Mz19-positive PNs (green in $\boldsymbol{A}$ ). The presynaptic partner (Or88a) axons remain stable as the distant control axons (compare blue staining in $\boldsymbol{B}$ with $\boldsymbol{A}$; or axon only channel in $\boldsymbol{B}^{\prime}$ with $\left.\boldsymbol{A}^{\prime}\right)$. $(-\boldsymbol{F}$, Control $(\boldsymbol{C}, \boldsymbol{E})$ and cell ablation with DTI $(\boldsymbol{D}, \boldsymbol{F})$ of Gal4-GH146-positive PNs (green in $\boldsymbol{C}, \boldsymbol{E}$ ). After their postsynaptic $P N$ partners are ablated, axons of three ORN classes as indicated (blue in $\boldsymbol{D}, \boldsymbol{F}$; or alone in $\boldsymbol{D}^{\prime}, \boldsymbol{F}^{\prime}$ ) remain intact compared with controls (blue in $C, E$; or alone in $C^{\prime}, E^{\prime}$ ). Green is mCD8GFP, red is nc82, and blue is CD2. $\boldsymbol{A}^{\prime}-\boldsymbol{F}^{\prime}$ shows $C D 2$ only channels for $\boldsymbol{A}-\boldsymbol{F}$. Note that variations in the mounting angles result in changes of distances between glomeruli in projections of $z$ stacks shown in different samples. Genotypes: w; Gal4-Mz19 UAS-mCD8GFP/tubGal80ts; Or88a-CD2 Or10a-CD2/TM6b (A), w; Gal4-Mz19 UAS-mCD8GFP/tubGal80ts; Or88a-CD2 Or10a-CD2/UAS-DTI (B), yw hs-Flp122 UAS-mCD8GFP/w; Gal4-GH146 UASmCD8GFP/tubGal80ts; Or88a-CD2 Or10a-CD2/TM6b (C), yw hs-Flp122 UAS-mCD8GFP/w; Gal4-GH146 UAS-mCD8GFP/tubGal80ts; Or88a-CD2 Or10a-CD2/UAS-DTI (D), yw hs-FIp122 UAS-mCD8GFP/w; Gal4-GH146 UAS-mCD8GFP/tubGal80ts; Or47b-CD2 Or10aCD2/TM6b (E), and yw hs-Flp122 UAS-mCD8GFP/w; Gal4-GH146 UAS-mCD8GFP/tubGal80ts; Or47b-CD2 Or10a-CD2/UAS-DTI (F). 

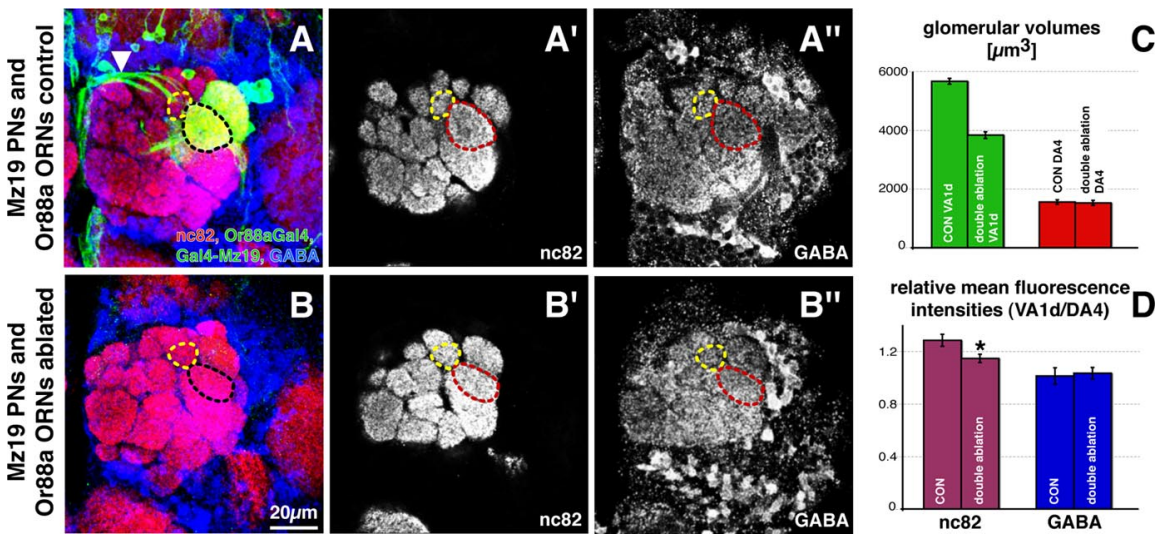

Figure 7. Double ablation of VA1d ORNs and PNs. $\boldsymbol{A}, \boldsymbol{B}$, Control $(\boldsymbol{A})$ and DTI ablation $(\boldsymbol{B})$ of Mz19 PNs and Or88a ORNs projecting to VA1d. Green in $\boldsymbol{A}$ shows GFP-labeled Or88a ORN axon terminals and MZ19 dendrites, both of which are ablated in experimental conditions $(\boldsymbol{B}) . \boldsymbol{A}^{\prime}$ and $\boldsymbol{B}^{\prime}$ show single confocal sections of the nc82 staining alone (magenta in $\boldsymbol{A}, \boldsymbol{B}$ ). $\boldsymbol{A}^{\prime \prime}$ and $\boldsymbol{B}^{\prime \prime}$ show the single confocal sections of GABA staining alone (blue in $\boldsymbol{A}, \boldsymbol{B}$ ). $\boldsymbol{C}$, Quantification of VA1d glomerular volumes in control (CON VA1d) and double-ablation (double-ablation VA1d) experiments (green), with DA4 as a control (red). $n=13$ for control, 12 for experiment. Error bars indicate SEM. D, Quantification of ratios of relative mean fluorescence intensities (number of pixels per measured area) between VA1d and DA4 for nc 82 (purple) and GABA (blue) stainings. We observe a slight but significant relative decrease in $n c 82$ staining in VA1d glomeruli without ORN and PN processes (double ablation) compared with the control (CON) $\left({ }^{*} p<0.02\right.$ in two-tailed $t$ test), whereas relative GABA levels are unchanged. $n=13$ for control, 12 for experiment. Error bars indicate SEM. Genotypes:yw hs-FIp UAS-mCD8GFP/w; Gal4-Mz19 UAS-mCD8GFP/Or88aGal4Mz19mCD8GFPy+; tubGal80ts/TM6b (A) (CON in C, D), and double ablation is yw hs-FIp UAS-m(D8GFP/w; Gal4-Mz19 UAS-m(D8GFP/Or88aGal4 Mz19m(D8GFPy+; UAS-DTI) tubGal80ts (B) (double ablation in $\boldsymbol{C}, \boldsymbol{D})$.

Remaining structures in the glomerulus could be contributed from either the remaining synaptic partners or processes from other cell types. To distinguish between these two possibilities, we simultaneously ablated ORNs and PNs projecting to the VA1d glomerulus (Fig. 7). Remarkably, the VA1d glomerulus was still readily distinguishable by the presynaptic nc82 marker staining (Fig. $7 B^{\prime}$, red circle). The size of the VA1d glomerulus was significantly smaller (compared with Fig. $7 A^{\prime}$; quantified in $C$ ), but this size reduction was similar in magnitude compared with ORN ablation alone (Fig. 2G). This experiment indicates that processes from other cell types contribute significantly to the volume of glomeruli.

Indeed, in addition to ORN axons and PN dendrites, the insect antennal lobe also contains a third, less characterized neuronal element: the dendrites of local interneurons (LNs). Each LN sends out profuse dendrites to innervate large areas of the antennal lobe without projecting axons to higher brain centers (Fig. $1 A$ ). They form widespread, inhibitory connections to PNs, other LNs, and ORN axons (Stocker, 1994; Ng et al., 2002). To test whether the residual cellular elements were contributed by LNs, we stained the antennal lobe with an anti-GABA antibody to visualize $\mathrm{LN}$ processes in the control and double-ablated glomerulus (Fig. $7 A^{\prime \prime}, B^{\prime \prime}$ ). LNs are known to be mostly inhibitory neurons whereas ORNs and PNs are excitatory, so GABA staining should selectively label LNs. Indeed, GABA-positive cell body distribution around the antennal lobe was consistent with what was known about the location of LNs (Ng et al., 2002). Consistent with the extensive distribution of LN dendrites, GABA immunoreactivity was found uniformly throughout the antennal lobe in controls (Fig. $7 A^{\prime \prime}$ ). As predicted, GABA immunoreactivity was present to a similar extent in the remaining VAld glomerulus after ORNs and PNs had been ablated (Fig. $7 B^{\prime \prime}, D$ ).

Studies from various insect species suggest that, within glomeruli, extensive synapses possibly occur between $\mathrm{ORN} \rightarrow \mathrm{PN}$, $\mathrm{ORN} \rightarrow \mathrm{LN}, \mathrm{PN} \rightarrow \mathrm{LN}, \mathrm{LN} \rightarrow \mathrm{PN}, \mathrm{LN} \rightarrow \mathrm{ORN}$, and $\mathrm{LN} \rightarrow \mathrm{LN}$ (Stocker, 1994; Distler and Boeckh, 1998; Ng et al., 2002). All but the last synaptic connections should be disrupted in the double-ablation condition of VAld. A priori, one might expect a severe reduction of synaptic density in VA1d. However, apart from the reduction of the glomerular volume, most likely caused by loss of ORN axons, the remaining glomerulus showed only a slight reduction of total presynaptic density as judged by nc82 staining in the doubleablation condition compared with the control (Fig. 7D). The overall maintenance of synaptic density did not appear to be caused by an increase in the density of LNs processes, because there was no change of the density of GABA immunoreactivity (Fig. 7D). A parsimonious explanation is that $\mathrm{LN}$ processes are a major contributor to the normal glomerular volume and LN-LN synapses contribute a significant portion of total synapses in glomeruli. The high LN cell number per antennal lobe $(\sim 100)$ (Stocker, 1994; $\mathrm{Ng}$ et al., 2002) and their capability to invade many if not all glomeruli make this explanation plausible. Because of the lack of available Gal4 lines that selectively label large subsets of antennal lobe-specific LNs, we were not able to experimentally test the contribution of LNs to the stability of the olfactory circuit at the antennal lobe.

\section{Intraglomerular plasticity after unilateral antenna removal}

The lack of wiring plasticity in our experiments so far could be explained by the inability of the Drosophila olfactory system to undergo any plastic changes. Alternatively, the laboratory conditions for rearing flies might prevent plasticity. However, the following experiment indicates that, under the same culture conditions, wiring plasticity in the adult olfactory system can occur, albeit restricted within a glomerulus.

Previous work demonstrated that most ORNs project their axons to both the ipsilateral and contralateral glomerulus of the same type in the left and right antennal lobes (Stocker et al., 1990). After unilateral deafferentation, the position and size of the glomerulus were unaffected, but the axon density was significantly decreased (Vosshall et al., 2000). Using this property of the Drosophila olfactory system, we tested whether ORN axons could adjust their axon terminal densities when ORN input from one side was removed.

We cut off the left third antennal segment of young adults and followed the change of fluorescence intensity of Or88a-Gal4driven mCD8GFP over time as a measure of axon density in the left and right VA1d glomerulus. As a built-in calibration for the fluorescence intensity measurement, we introduced in the same animal the maxillary palp-specific Or46a-Gal4 driver to label projections to VA7l, which is unaffected by the antenna removal (Fig. 8A-D, green) (see also Fig. 4). In the control, antennae are intact and projections in VAld and VA7l are identical for both antennal lobes (Fig. $8 A, E$ ). After both antennae are cut off, VA1d axons cannot be detected any more in both glomeruli after $3 \mathrm{~d}$, whereas axons in VA7l remain unchanged (Fig. $8 \mathrm{~B}$ ). However, $3 \mathrm{~d}$ after, only the left antenna was removed (by this time, all VA1d axons originating from the left antenna should have degenerated); we detected a twofold difference in fluorescence intensity 
between the left and right antennal lobes. The simplest explanation for this difference is that, in normal animals, Or88a ORNs have twice as many axon terminal arborizations in the ipsilateral as contralateral VAld glomerulus, assuming that no plastic changes occur within the first $3 \mathrm{~d}$ after unilateral denervation (but see below).

Interestingly, $7 \mathrm{~d}$ after unilateral antenna removal, this twofold asymmetry in relative fluorescence intensity is significantly reduced to 1.5 -fold (Fig. 8, compare green signal within white circles in $C, D$; quantified in $E$ ). This result indicates that ORNs readjust the densities of their axon arborizations when competing input from ORNs from the other side is removed. However, this plasticity is confined within the glomerulus, because Or88a-positive axon terminals are confined within its appropriate VAld glomerulus.

The decrease in the ratio of relative fluorescence intensity between the left and right VAld glomerulus over time could result from increased arborization of the contralateral or reduced arborization of the ipsilateral axons from the intact antenna, or both. We compared the relative fluorescence intensities (VAld/VA7l) of the left and right VA1d glomerulus between 3 and $7 \mathrm{~d}$ after left, unilateral deafferentation. In the left antennal lobe, a significant increase in VAld fluorescence intensity was detected at $7 \mathrm{~d}$, whereas in the right antennal lobe, the VA1d fluorescence intensity is similar at 3 and $7 \mathrm{~d}$ (Fig. $8 F)$. This suggests that intraglomerular plasticity is mostly contributed by increased terminal arborizations of contralateral projections.

We also measured the axon densities 10 and $14 \mathrm{~d}$ after unilateral antenna removal. The 1.5 -fold asymmetry was not reduced further (Fig. $8 E$ ). This could be because either intraglomerular plasticity can only occur during a limited time window in young adults or equality cannot be reached because of cell biological constraints: for instance, contralateral branches need to be maintained by ORN axonal transport of longer distances. The latter may explain why axon terminal arborizations seem twice as dense in the ipsilateral as contralateral glomerulus in normal animals. The twofold difference, assessed $3 \mathrm{~d}$ after unilateral denervation, is likely an underestimate, because intraglomerular plasticity could already occur within $3 \mathrm{~d}$ after antennal removal.

In summary, we show that intraglomerular plasticity in VA1d occurs between 3 and $7 \mathrm{~d}$ after unilateral antenna removal and is likely caused by an increased arborization of contralateral projections. Therefore, we conclude that the olfactory circuit can exhibit plasticity under our experimental conditions. However, the plasticity is confined within an individual glomerulus.

\section{Discussion}

Stability of the olfactory circuit

We used a genetic approach using cell-type-specific promoters driving toxin expression to ablate defined presynaptic and
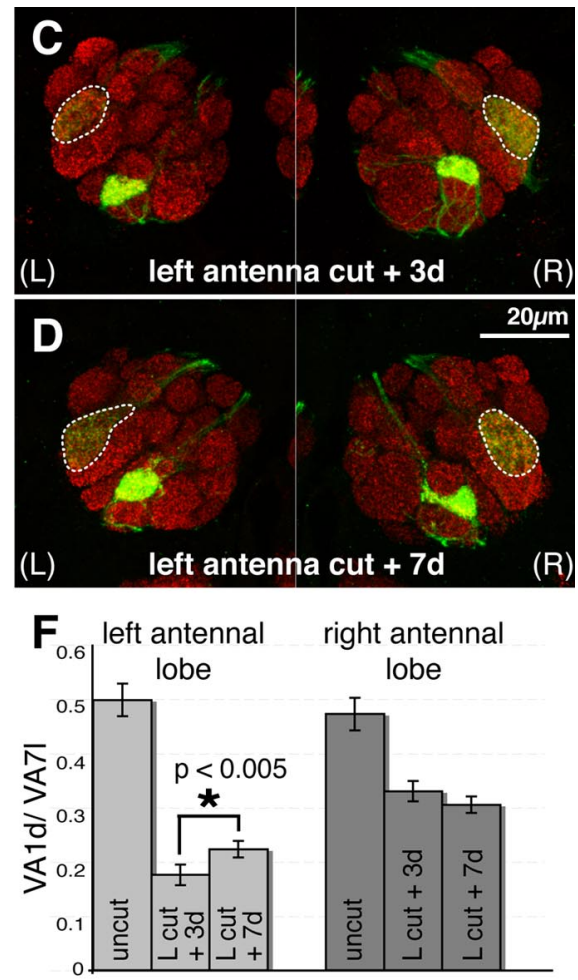

Figure 8. Intraglomerular axonal plasticity after unilateral antenna removal. $\boldsymbol{A}-\boldsymbol{D}$, Left $(\mathrm{L})$ and right $(\mathrm{R})$ antennal lobes in (A), 3 d after cutting both antennae $(\boldsymbol{B})$, and $3 \mathrm{~d}(\boldsymbol{C})$ or $7 \mathrm{~d}(\boldsymbol{D})$ after cutting only the left antenna. Or88a-positive axons from re labeled in are compared on the same side of the brain between 3 and $7 \mathrm{~d}$ after left antenna removal. $n=10,23$, and 31 for control, left antenna cut after 3 , and left antenna cut after $7 d$, respectively. Error bars indicate SEM. ${ }^{*} p<0.005$ in two-tailed $t$ test. Genotype: yw hs-Flp UAS-mCD8GFP/w; Or88aGal4/0r46aGal4 (A-F).

postsynaptic neuronal classes in the adult Drosophila olfactory circuit to examine the extent of its wiring plasticity. A similar approach has been used previously to ablate a specific class of mouse olfactory sensory neurons (Gogos et al., 2000) to answer a different question: do axons of sensory neurons born during adulthood rely on existing axons of the same class to reach their glomerular target? In mice, olfactory sensory neurons continuously renew throughout adult life. In contrast, fly ORNs are born only during a defined window of pupal development and are used throughout the fly's short life. Because ORNs are not replenished in the adult fly, we could study possible wiring reorganization after complete removal of a sensory neuron class. We were also able to specifically ablate certain classes of postsynaptic neurons in this circuit and to monitor the consequences on their presynaptic partners. Our general finding is that the adult olfactory circuit in the antennal lobe is extremely stable.

Before our study, several reports suggested the stability of different aspects of the olfactory circuit. In Drosophila, removing the entire population of ORNs does not significantly alter the axonal projections of PNs in higher brain centers when examined at a single-cell level (Wong et al., 2002) or in small groups (Tanaka et al., 2004). The gross connectivity of PNs with third-order neurons is not altered either (Tanaka et al., 2004). In mice, an in vivo imaging study in the mouse olfactory bulb showed remarkable stability of the apical dendrites of mitral/tufted (M/T) cells, the 
equivalent of Drosophila PNs. M/T cell dendrites in the glomeruli receive presynaptic input from ORNs and periglomerular cells, both of which turnover throughout life (Altman, 1969; MackaySim and Kittel, 1991). Despite the death and birth of their synaptic partners, $\mathrm{M} / \mathrm{T}$ cell dendritic branching patterns remain highly stable; this stability is also not affected by olfactory learning (Mizrahi and Katz, 2003).

Our study differs from these previous studies in its focus on examining the cross-wiring between different information channels. This is made possible by the glomerular organization of the Drosophila antennal lobe, in which individual classes of ORN axons make specific connections with individual $\mathrm{PN}$ dendrites at stereotypical glomeruli, and our ability to ablate specific classes of these channels either presynaptically (ORNs) or postsynaptically (PNs) using genetic tools. The majority of the experiments in our study is based on the ablation of specific classes of neurons, which could be more effective in generating changes compared with large-scale perturbations (Zhao and Reed, 2001; Yu et al., 2004). Thus, our study is conceptually similar to lesion studies in mammalian somatosensory or visual systems, in which profound wiring reorganization was reported (see Introduction). However, the results of all neuronal ablation experiments in the adult Drosophila olfactory system we performed support a common theme: when synaptic partners or neighbors have been eliminated, ORN or PN processes do not sprout to neighboring glomeruli and therefore wiring specificity is not altered.

Why do our findings differ from previous work in mammals on lesion-induced neuronal wiring? One possibility is that neuronal circuits in Drosophila (and perhaps invertebrates in general) are inherently much more hardwired and not adaptable to changes. Although little has been reported on injury-induced wiring plasticity in adult Drosophila, developmental wiring plasticity can certainly occur. For example, the stereotypic neuromuscular connections in Drosophila larvae are able to reorganize after the ablation of a specific motoneuron during development. The corresponding target muscle can receive synaptic input from neighboring motoneurons as a result of axonal sprouting of neighboring motor neurons (Chang and Keshishian, 1996). Likewise in the Drosophila visual system, photoreceptor axons can sprout into neighboring empty space created by a cell fate transformation (Ashley and Katz, 1994). In addition, significant changes of neuropil volumes in the adult Drosophila brain in response to sensory experience have been reported (Heisenberg et al., 1995; Devaud et al., 2001).

Importantly, we show directly that unilateral deafferentation of antennal ORNs leads to a significant increase of axon density of contralateral projections from the intact antenna (Fig. 8). This finding indicates that ORN axons are capable of altering their axon terminal arborizations when competing axons are removed and that the Drosophila adult olfactory circuit can adapt to changes under our culture conditions. However, such plasticity is restricted within an individual glomerulus.

\section{Continuous versus discrete neuronal maps}

A fundamental difference of this study and previous studies on lesion-induced wiring plasticity is the nature of the neural maps. Whereas most previous studies focus on systems with a continuous map (e.g., somatosensory and visual systems), the olfactory map is discrete. As in mammals, most Drosophila olfactory receptor neurons express a single, specific olfactory receptor, and ORNs expressing the same receptor converge onto the same glomerulus in the antennal lobe, creating discrete channels for information processing. Thus, unlike the visual or somatosensory system in which neighboring neurons represent neighboring sensory space, neighboring glomeruli in the olfactory system may not necessarily represent neighboring odor space or convey similar biological values.

The olfactory circuit may be built to ensure the discreteness of these information channels. In Drosophila, the establishment of wiring specificity of the olfactory circuit is primarily achieved before the onset of sensory experience, as judged by detectable OR expression or maturation of synaptic specialization (Jefferis et al., 2004 and references therein). The current study further shows that, in adults, this circuit is extremely stable in response to ablation of specific neuronal elements. The developmental hardwiring coupled with the maintenance of wiring specificity in adults may be essential for the olfactory system to mediate innate behaviors such as detecting food, predators, or mating partners. Indeed, unknown to us when we initiated this study, two of the three glomeruli (DA1 and VA1lm but not the intervening VA1d) we focus on are likely used for perception of sex pheromones during male courtship (Manoli et al., 2005; Stockinger et al., 2005). One can imagine that reorganization of neuronal wiring in response to injury, causing the muddling of information channels, may be more harmful for the fly than simply losing a single information channel.

Two properties of the olfactory circuit could contribute to wiring stability in the antennal lobe. First, each glomerulus in adults is wrapped by glial processes (Jefferis et al., 2004), which could prevent wiring plasticity by inhibiting axonal or dendritic sprouting across the glomerular border. Second, in addition to ORN axons and PN dendrites, dendrites of LNs also contribute significantly to the synaptic architecture of the glomeruli (Fig. 7). Although dendrites of each LN cover many, if not all, glomeruli and therefore do not represent channel-specific information, they are also postsynaptic targets of ORNs and may form reciprocal connections with PNs (Distler and Boeckh, 1998). Thus, after ablation of ORNs or PNs, the remaining partners can still retain synaptic connections with LNs. Even when ORNs and PNs are ablated simultaneously, the glomerulus still remains and maintains similar levels of GABA staining and close to normal levels of presynaptic terminals (Fig. 7), suggesting that a rich network of LN dendrites contributes significantly to the stability of the glomeruli.

Our results do not rule out the possibility of functional plasticity in the absence of structural changes. For instance, in the case of ORN ablation, the corresponding PN partners could represent other olfactory information through their remaining connections with LNs. Our results also do not exclude the possibility that wiring plasticity could still occur in higher olfactory centers when single input channels are removed from the periphery. It will be intriguing in the future to determine whether spatial maps in higher olfactory centers are plastic.

In summary, our results suggest that the balance between plasticity and stability of neuronal circuits, as assayed by lesioninduced structural changes, may differ for neuronal circuits that serve different functions. It will be important to investigate not only the mechanisms underlying neuronal plasticity and stability but also how their balance is regulated in different neuronal circuits.

\section{References}

Altman J (1969) Autoradiographic and histological studies of postnatal neurogenesis. IV. Cell proliferation and migration in the anterior forebrain, with special reference to persisting neurogenesis in the olfactory bulb. J Comp Neurol 137:433-457. 
Ashley JA, Katz FN (1994) Competition and position-dependent targeting in the development of the Drosophila R7 visual projections. Development 120:1537-1547.

Axel R (1995) The molecular logic of smell. Sci Am 273:154-159.

Buonomano DV, Merzenich MM (1998) Cortical plasticity: from synapses to maps. Annu Rev Neurosci 21:149-186.

Chang TN, Keshishian H (1996) Laser ablation of Drosophila embryonic motoneurons causes ectopic innervation of target muscle fibers. J Neurosci 16:5715-5726.

Clyne PJ, Warr CG, Freeman MR, Lessing D, Kim J, Carlson JR (1999) A novel family of divergent seven-transmembrane proteins: candidate odorant receptors in Drosophila. Neuron 22:327-338.

Couto A, Alenius M, Dickson BJ (2005) Molecular, anatomical, and functional organization of the Drosophila olfactory system. Curr Biol 15:1535-1547.

Darian-Smith C (2004) Primary afferent terminal sprouting after a cervical dorsal rootlet section in the macaque monkey. J Comp Neurol 470:134-150.

Darian-Smith C, Brown S (2000) Functional changes at periphery and cortex following dorsal root lesions in adult monkeys. Nat Neurosci $3: 476-481$

Darian-Smith C, Gilbert CD (1994) Axonal sprouting accompanies functional reorganization in adult cat striate cortex. Nature 368:737-740.

Devaud JM, Acebes A, Ferrus A (2001) Odor exposure causes central adaptation and morphological changes in selected olfactory glomeruli in Drosophila. J Neurosci 21:6274-6282.

Distler PG, Boeckh J (1998) An improved model of the synaptic organization of insect olfactory glomeruli. Ann NY Acad Sci 855:508-510.

Dunin-Borkowski OM, Brown NH (1995) Mammalian CD2 is an effective heterologous marker of the cell surface in Drosophila. Dev Biol 168:689-693.

Fishilevich E, Vosshall LB (2005) Genetic and functional subdivision of the Drosophila antennal lobe. Curr Biol 15:1548-1553.

Florence SL, Taub HB, Kaas JH (1998) Large-scale sprouting of cortical connections after peripheral injury in adult macaque monkeys. Science 282:1117-1121.

Gao Q, Chess A (1999) Identification of candidate Drosophila olfactory receptors from genomic DNA sequence. Genomics 60:31-39.

Gao Q, Yuan B, Chess A (2000) Convergent projections of Drosophila olfactory neurons to specific glomeruli in the antennal lobe. Nat Neurosci 3:780-785.

Gerlitz O, Nellen D, Ottiger M, Basler K (2002) A screen for genes expressed in Drosophila imaginal discs. Int J Dev Biol 46:173-176.

Gilbert CD, Wiesel TN (1992) Receptive field dynamics in adult primary visual cortex. Nature 356:150-152.

Gogos JA, Osborne J, Nemes A, Mendelsohn M, Axel R (2000) Genetic ablation and restoration of the olfactory topographic map. Cell 103:609-620.

Goldman AL, Van der Goes van Naters W, Lessing D, Warr CG, Carlson JR (2005) Coexpression of two functional odor receptors in one neuron. Neuron 45:661-666

Han DD, Stein D, Stevens LM (2000) Investigating the function of follicular subpopulations during Drosophila oogenesis through hormonedependent enhancer-targeted cell ablation. Development 127:573-583.

Heisenberg M, Heusipp M, Wanke C (1995) Structural plasticity in the Drosophila brain. J Neurosci 15:1951-1960.

Hickmott PW, Steen PA (2005) Large-scale changes in dendritic structure during reorganization of adult somatosensory cortex. Nat Neurosci $8: 140-142$

Jefferis GS, Marin EC, Stocker RF, Luo L (2001) Target neuron prespecification in the olfactory map of Drosophila. Nature 414:204-208.

Jefferis GS, Vyas RM, Berdnik D, Ramaekers A, Stocker RF, Tanaka NK, Ito K, Luo L (2004) Developmental origin of wiring specificity in the olfactory system of Drosophila. Development 131:117-130.

Kaas JH (1991) Plasticity of sensory and motor maps in adult mammals. Annu Rev Neurosci 14:137-167.

Komiyama T, Carlson JR, Luo L (2004) Olfactory receptor neuron axon targeting: intrinsic transcriptional control and hierarchical interactions. Nat Neurosci 7:819-825.
Laissue PP, Reiter C, Hiesinger PR, Halter S, Fischback KF, Stocker RF (1999) Three-dimensional reconstruction of the antennal lobe in Drosophila melanogaster. J Comp Neurol 405:543-552.

Lund JP, Sun GD, Lamarre Y (1994) Cortical reorganization and deafferentation in adult macaques. Science 265:546-548.

Mackay-Sim A, Kittel P (1991) Cell dynamics in the adult mouse olfactory epithelium: a quantitative autoradiographic study. J Neurosci 11:979-984.

Manoli DS, Foss M, Villella A, Taylor BJ, Hall JC, Baker BS (2005) Malespecific fruitless specifies the neural substrates of Drosophila courtship behaviour. Nature 436:395-400

McGuire SE, Mao Z, Davis RL (2004) Spatiotemporal gene expression targeting with the TARGET and gene-switch systems in Drosophila. Sci STKE 2004:pl6.

Mizrahi A, Katz LC (2003) Dendritic stability in the adult olfactory bulb. Nat Neurosci 6:1201-1207.

Ng M, Roorda RD, Lima SQ, Zemelman BV, Morcillo P, Miesenbock G (2002) Transmission of olfactory information between three populations of neurons in the antennal lobe of the fly. Neuron 36:463-474.

Pons TP, Garraghty PE, Ommaya AK, Kaas JH, Taub E, Mishkin M (1991) Massive cortical reorganization after sensory deafferentation in adult macaques. Science 252:1857-1860.

Robinson M, Ranjan R, Schwarz TL (2002) Synaptotagmins I and IV promote transmitter release independently of $\mathrm{Ca}^{2+}$ binding in the $\mathrm{C}(2) \mathrm{A}$ domain. Nature 418:336-340.

Sepp KJ, Auld VJ (1999) Conversion of lacZ enhancer trap lines to GAL4 lines using targeted transposition in Drosophila melanogaster. Genetics 151:1093-1101.

Smirnakis SM, Brewer AA, Schmid MC, Tolias AS, Schuz A, Augath M, Inhoffen W, Wandell BA, Logothetis NK (2005) Lack of long-term cortical reorganization after macaque retinal lesions. Nature 435:300-307.

Smith HK, Roberts IJH, Allen MJ, Connolly JB, Moffat KG, O'Kane CJ (1996) Inducible ternary control of transgene expression and cell ablation in Drosophila. Dev Genes Evol 206:14-24.

Stocker RF (1994) The organization of the chemosensory system in Drosophila melanogaster: a review. Cell Tissue Res 275:3-26.

Stocker RF, Lienhard MC, Borst A, Fischbach K-F (1990) Neuronal architecture of the antennal lobe in Drosophila melanogaster. Cell Tissue Res 262:9-34.

Stocker RF, Heimbeck G, Gendre N, de Belle JS (1997) Neuroblast ablation in Drosophila P[GAL4] lines reveals origins of olfactory interneurons. J Neurobiol 32:443-456.

Stockinger P, Kvitsiani D, Rotkopf S, Tirian L, Dickson BJ (2005) Neural circuitry that governs Drosophila male courtship behavior. Cell 121:795-807.

Tanaka NK, Awasaki T, Shimada T, Ito K (2004) Integration of chemosensory pathways in the Drosophila second-order olfactory centers. Curr Biol 14:449-457.

Vosshall LB (2000) Olfaction in Drosophila. Curr Opin Neurobiol 10:498-503.

Vosshall LB, Amrein H, Morozov PS, Rzhetsky A, Axel R (1999) A spatial map of olfactory receptor expression in the Drosophila antenna. Cell 96:725-736.

Vosshall LB, Wong AM, Axel R (2000) An olfactory sensory map in the fly brain. Cell 102:147-159.

Wiesel TN (1982) Postnatal development of the visual cortex and the influence of environment. Nature 299:583-591.

Wong AM, Wang JW, Axel R (2002) Spatial representation of the glomerular map in the Drosophila protocerebrum. Cell 109:229-241.

Yu CR, Power J, Barnea G, O’Donnell S, Brown HE, Osborne J, Axel R, Gogos JA (2004) Spontaneous neural activity is required for the establishment and maintenance of the olfactory sensory map. Neuron 42:553-566.

Zhao H, Reed RR (2001) X inactivation of the OCNC1 channel gene reveals a role for activity-dependent competition in the olfactory system. Cell 104:651-660.

Zhu H, Luo L (2004) Diverse functions of N-cadherin in dendritic and axonal terminal arborization of olfactory projection neurons. Neuron 42:63-75. 